\title{
Predictive value of frailty on postoperative complications in elderly patients with major abdominal surgery.
}

\author{
Binru Han*, Yanqiu Wang, Xi Chen \\ Xuanwu Hospital, Capital Medical University, PR China
}

\begin{abstract}
Aims: To investigate the predictive value of frailty on postoperative complications in elderly patients undergoing major abdominal surgery.

Study design: This was a prospective cohort study.

Methods: Frailty phenotype assessment instruments were applied to evaluate the frailty of patients, and a self-designed questionnaire was employed to collect disease related data, including general demographics, a physiological index POSSUM score and an operative severity index. Postoperative complications were recorded during clinical follow-up. Differences in complications between groups were analysed to determine whether frailty was an independent influencing factor for postoperative complications in elderly patients undergoing abdominal surgery and to determine the predictive value of frailty.

Results: The incidence of frailty in elderly patients undergoing abdominal surgery was $23.74 \%$ ( $n=176$ ). The incidence of postoperative complications in the frailty group was $50 \%(\mathrm{n}=\mathbf{3 2})$, significantly higher than that in the non-frailty group $(7.64 \%, n=144 ; \mathbf{p}<0.0001)$. Frailty was an independent influencing factor for postoperative complications in elderly patients undergoing major abdominal surgery. The area under the curve of frailty in the prediction of postoperative complications in elderly patients with major abdominal surgery was $\mathbf{0 . 7 5 7}$, indicating that frailty had the same predictive value as POSSUM. Conclusion: Frailty is an effective predictor for the occurrence of postoperative complications in elderly patients undergoing major abdominal surgery.

Relevance to clinical practice: Frailty is related to the occurrence of postoperative complications to assess the surgical risk of patients. Frailty assessment provides a new tool for nurses involved with the preoperative care and risk assessment of elderly patients with surgical risk.
\end{abstract}

Keywords: Surgery, Preoperative, Nursing assessment, Older patients.

Accepted on January 13, 2018

\section{Introduction}

Surgical intervention is a conventional treatment method. According to annual statistical data from the National Bureau of Statistics, 43,829,200 inpatients underwent surgical treatment in hospitals and medical institutions in 2014, accounting for $21.44 \%$ of all inpatients that year [1]. With the growth of aging populations throughout the world, the elderly have become the main population admitted to medical institutions. Medical statistics from the USA indicate that more than $1 / 3$ of the patients who undergo surgical treatment are over 65 y old [2]. Overall adaptation and tolerance in elderly patients are decreased because of higher levels of complications and delayed onset of disease. The organs of the elderly are susceptible to decompensation during emergencies, especially when combined with stress events, such as hospitalization or surgery, which leads to postoperative complications. Postoperative complication is the main factor influencing postoperative prognosis. Therefore, a comprehensive and accurate preoperative risk assessment for patients plays an important role in the prediction of adverse outcomes and in the decision-making process.

\section{Background}

Major abdominal surgery is a significant stressor for the elderly, leading to endocrine, metabolic and immune dysfunction, subsequent physiological changes and a decreased quality of life [3]. Approximately $35 \%$ of patients experience postoperative complications and the $30 \mathrm{~d}$ case fatality rate is approximately $10 \%$ [4]. Postoperative complications may initiate cascading reactions, such as disability, inability to manage self-care, decreased quality of life, increased medical costs and other adverse outcomes [5]. The existing clinical preoperative risk assessment instruments, such as Glasgow, POSSUM (the Physiological and Operative Severity Score for the Enumeration of Mortality and Morbidity), ASA (American Association of Anesthesiologists) and other assessment tools for specific organs or systems (such as Goldman), are aimed at the characteristics of the operation or the disease itself and fail 
to take the physical condition of the patients into account. With increasing gage, the probability of body abnormalities increases due to age-related changes [6]. However, individual aging is characterized by heterogeneity [7], necessitating health assessment methods designed specifically for elderly patients, such as the CGA (comprehensive geriatric assessment) and assessments of sarcopenia and frailty [8]. The CGA assessment requires multidisciplinary teams and multi-dimensional evaluations [9], which are significant obstacles in clinical practice. In 2012, experts from six international professional associations reached a consensus definition of frailty, i.e., frailty is a clinical syndrome caused by multiple factors, with the main characteristics of decreased strength and endurance and reduced physiological tolerance. Frailty in individuals enhances their susceptibility to disability or death, and frailty screening is recommended for all individuals over $70 \mathrm{y}$ old [10]. The American College of Surgeons recommends that frailty assessment be implemented in preoperative risk evaluations [11]. Researchers believe that physiological status is more related to the occurrence of postoperative death than surgery [12]. Therefore, this prospective cohort study was designed to investigate the effects of frailty on postoperative complications in elderly patients undergoing major abdominal surgery to provide a new method of clinical care for preoperative risk management.

\section{Methods}

\section{Participants}

Our study was approved by the Xuanwu Hospital Capital Medical University medical quality and safety management in January 2015. A prospective cohort from February-October 2015 was used to investigate the predictive value of frailty on postoperative complications in elderly patients undergoing major abdominal surgery. The survey data were provided anonymous codes to ensure that identifying information remained confidential. A total of 176 patients were enrolled in the study.

\section{The inclusion and exclusion criteria for recruiting the participants}

Inclusion criteria: 1 . Age $\geq 60 \mathrm{y} ; 2$. being admitted to department of surgery due to abdominal digestive diseases; stable disease; 3 . being scheduled for elective surgery; 4. willingness to participate in this study.

Exclusion criteria: 1. History of Parkinson's disease or stroke; currently taking carbidopa, levodopa, donepezil hydrochloride and/or antidepressants, as they may produce the symptoms similar to emaciation; 2. cognitive impairments or psychiatric disorders; 3. having not undergone any major abdominal surgeries; 4. hypothyroidism; 5. language impairments preventing the patient from understanding the questions asked.

\section{Investigative tool}

General information: General information including age, sex, BMI, smoking and drinking history, comorbidity and laboratory indexes was recorded. BMI was calculated as height/body weight ${ }^{2}$, and comorbidity was assessed using the CCI scale.

Frailty assessment: The frailty phenotype proposed by Dr. Fried following the cardiovascular health study includes five indicators: unintentional weight loss, self-reported exhaustion, low activity level, slow walking speed and weak grip strength. One point is scored for each indicator when a specific target is reached. Subjects scoring 0 were classified as robust, 1-2 as pre-frail and 3-5 as frail.

The five indicators were operationalized as follows:

1. "Weight loss" was defined as self-reported un-intentional weight loss of more than $3 \mathrm{~kg}$ or greater than $5 \%$ of body weight during the previous year.

2. "Exhaustion" was indicated by a self-response as "a moderate amount the time" or "most of the time" to either of the following statements: "I felt everything I did was an effort" or "I could not get going" based on the Center for Epidemiological Studies-Depression Scale.

3. "Low physical activity" was defined by gender specific low weekly energy expenditures measured by the IPAQ-SF (International Physical Activity Questionnaire-Short Form).

4. "Slow walking speed" was defined as a usual walking speed below the criterion-specific thresholds adjusting for gender and height from the Cardiovascular Health Study Group.

5. "Weakness" was defined as mean grip strength of the dominant hand that was three times below the criterion-specific thresholds adjusting for gender and body mass index (BMI) defined in the Cardiovascular Health Study Group.

POSSUM scoring system: POSSUM was proposed by the British scholar Copeland [13], in 1991 based on data from general surgical operation cases. It can be used to predict the incidence of postoperative complications and death. This system includes twelve independent preoperative physiological factors (age, heart signs, respiratory signs, systolic blood pressure, pulse rate, Glasgow coma score, hemoglobin, white blood cells, blood urea nitrogen, sodium, potassium and ECG) and six operative severity indexes (surgical margin, operation number, total blood loss, abdominal contamination and malignant tumor). It is mainly applicable to general surgery.

\section{Postoperative complications}

Postoperative complications that occurred in patients during hospitalization were used to measure out comes in this study. Complications were categorized according to the common postoperative complications defined in the National Surgical Quality Improvement Program (NSQIP) by the American College of Surgeons (ACS) [14]. 


\section{Statistical analysis}

Data were recorded using Epidata 3.1 and statistically analysed by SPSS 17.0 software. Measurement data were expressed as the mean \pm standard deviation or median and four percentile. Comparisons between two in dependent samples used at-test and the Mann-Whitney $U$ test was used for comparisons between two groups. Enumeration data were presented as percentages, and the chi squared test/continuous correction chi squared test/Fisher exact probability test was applied for comparisons between the two groups. A CMH stratified chi squared test was employed to adjust for confounding factors. Factors influencing postoperative complications were analysed by a logistic regression based on the maximum likelihood method. The Receiver Operator Characteristic curve (ROC) was plotted, and the Area under the Curve (AUC) was calculated to determine the predictive values of frailty and POSSUM for postoperative complications. A P-value of $<0.05$ was considered statistically significant, and any missing data were deleted from the analysis.

\section{Results}

Of the 287 patients enrolled in this study, 176 met the inclusion criteria for this study. The mean age was $69.50 \pm 7.55 \mathrm{y}$. The frailty group included 32 patients and the non-frailty group included 144 patients.

Of the 176 patients that underwent major surgery, there were 32 cases with frailty and 144 without frailty (including 92 cases with pre-frailty, accounting for $52.27 \%$ of all patients). The incidence rate of frailty in patients with major abdominal surgery was $18.18 \%$. Postoperative complications occurred in 27 patients (15.34\%). There was a statistically significant difference in the incidence of postoperative complications between the frailty group $(50 \%)$ and the non-frailty group (7.64\%) (RR=12.091, 95\% CI: 4.768-0.536, Table 1).

A univariate analysis of the basic characteristics of the two groups was conducted to adjust for confounding factors. Results showed statistically significant differences between the two groups in age, $\mathrm{CCI}$, preoperative $\mathrm{ALB}, \mathrm{HB}, \mathrm{WBC}$, and $\mathrm{Na}$ + levels (Table 2). When these variables were used for stratification, differences in the rates of postoperative complications between the two groups remained statistically significant as determined by a stratified chi squared test $(\mathrm{RR}=6.625-12.955)$ (Table 3).

\section{Relationships between frailty and postoperative complications in elderly patients undergoing major abdominal surgery}

Using the physiological index from POSSUM and the surgical invasion index, postoperative complications were compared between the complication group and the non-complication group by univariate analysis (Table 4). Using variables that showed significant differences between the two groups (age, pulse rate, hemoglobin, $\mathrm{Na}^{+}$, the extent of surgery, blood loss, abdominal contamination and malignant tumor), frailty as an independent variable and postoperative complications as the dependent variable, logistic (maximum likelihood) regression analysis showed that frailty was an independent risk factor for postoperative complications in elderly patients that had undergone major abdominal surgery (adjusted $\mathrm{RR}=6.415$, Tables 5 and 6).

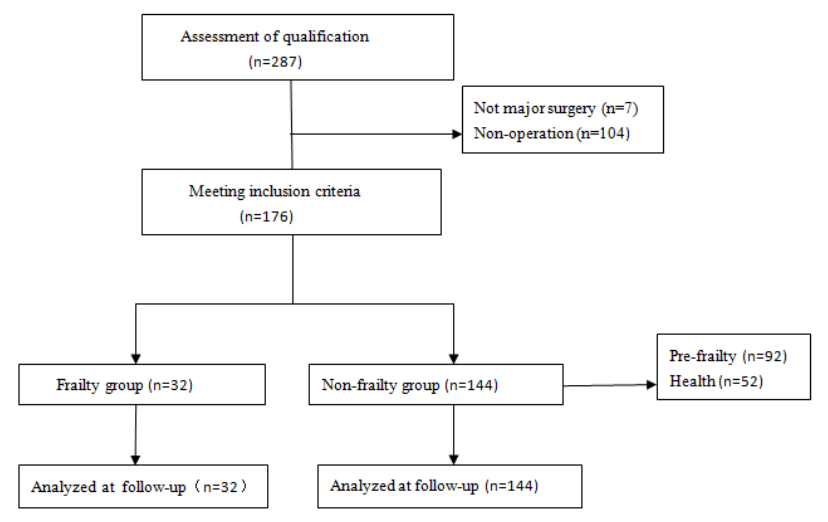

Figure 1. Prospective cohort trial design.

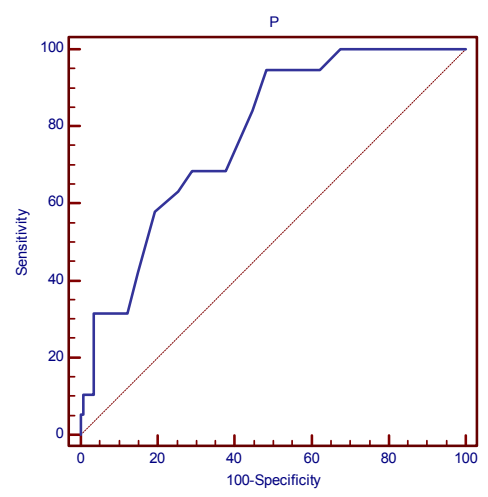

Figure 2. ROC curves of POSSUM for postoperative complications.

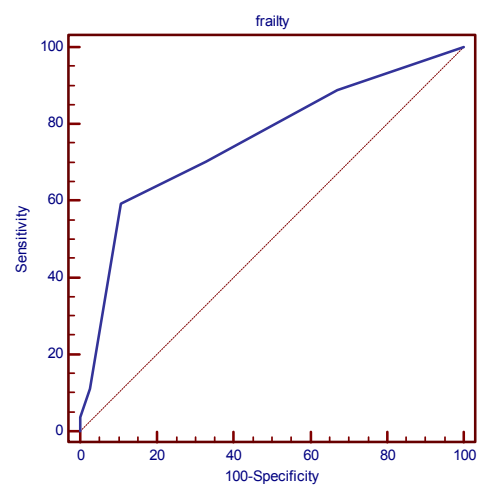

Figure 3. ROC curves of frailty for postoperative complications.

\section{The predictive value of frailty for postoperative complications in elderly patients that underwent major abdominal surgery}

The ROC curve was used to determine the predictive value of frailty for postoperative complications after major abdominal surgery. Results show that the AUCs for frailty and POSSUM 
were 0.757 and 0.777 , respectively, which are medium-level predictive values. The AUCs of the two factors were compared $(\mathrm{Z}=1.226, \quad \mathrm{P}=0.2203>0.05)$, and the difference was not statistically significant (Table 7, Figures 1-3). Therefore, our conclusion was that frailty and POSSUM shared the same predictive values.

Table 1. Comparison of postoperative complications in elderly patients undergoing major abdominal surgery between the two groups $(n=176)$.

\begin{tabular}{lllll}
\hline Frailty level & $\begin{array}{l}\text { Case numberl } \\
\text { proportion }\end{array}$ & $\begin{array}{l}\text { Postoperative } \\
\text { complications/ } \\
\text { incidence }\end{array}$ & $x^{2}$ & $p$
\end{tabular}

\begin{tabular}{lllll}
\hline Non-frailty & $144(81.82 \%)$ & $11(7.64 \%)$ & $\begin{array}{l}32.987 \\
1\end{array}$ & $<0.0001$ \\
\hline Frailty & $32(18.18 \%)$ & $16(50 \%)$ & & \\
\hline Health & $52(29.55 \%)$ & $3(5.77 \%)$ & 24.324 & $<0.0001$ \\
& & & 2 & \\
\hline Pre-frailty & $92(52.27 \%)$ & $8(8.93 \%)$ & & \\
\hline Frailty & $32(18.18 \%)$ & $16(50 \%)$ & & \\
\hline
\end{tabular}

Table 2. Comparison of basic data between the frailty group and the non-frailty group.

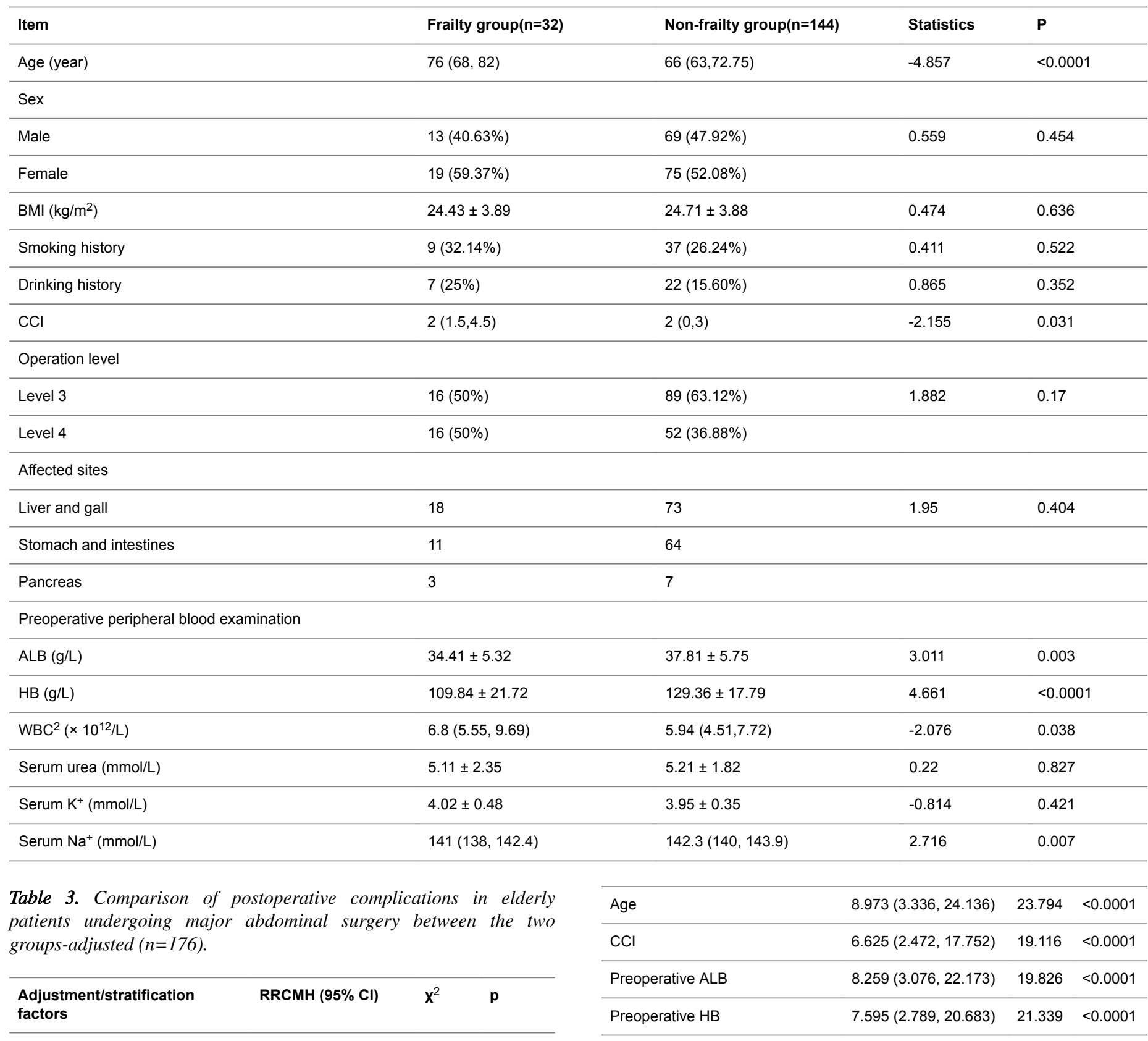


Predictive value of frailty on postoperative complications in elderly patients with major abdominal surgery

Preoperative WBC

$12.955(4.981,33.695) \quad 36.692<0.0001$

Preoperative $\mathrm{Na}^{+}$

$10.725(3.948,29.139) \quad 26.742<0.0001$

Table 4. Data comparison between the complication group and the non-complication group.

\begin{tabular}{|c|c|c|c|c|}
\hline Variables & Complication group & Non-complication group & Statistics & $\mathbf{p}$ \\
\hline Age & $75(65,82)$ & $67(63,73)$ & -3.137 & 0.002 \\
\hline \multicolumn{5}{|l|}{ Cardiac sign } \\
\hline No & 12 & 68 & 0.055 & 0.815 \\
\hline Abnormal & 15 & 77 & & \\
\hline \multicolumn{5}{|l|}{ Respiratory sign 4} \\
\hline No shortness of breath & 24 & 129 & $<0.0001$ & 0.991 \\
\hline Abnormal & 3 & 16 & & \\
\hline \multicolumn{5}{|l|}{ Systolic pressure $(\mathrm{mmHg})$} \\
\hline $110-130$ & 16 & 97 & 0.066 & 0.798 \\
\hline Other & 8 & 43 & & \\
\hline \multicolumn{5}{|l|}{ Pulse rate } \\
\hline $50-80$ & 17 & 128 & 7.371 & 0.007 \\
\hline Other & 7 & 11 & & \\
\hline \multicolumn{5}{|l|}{$\mathrm{HB}^{2}(\mathrm{~g} / \mathrm{L})$} \\
\hline $13-16$ & 8 & 57 & 16.018 & 0.001 \\
\hline $11.5-12.9 / 16.1-17.0$ & 5 & 54 & & \\
\hline $10.1-11.4 / 17.1-18.0$ & 5 & 24 & & \\
\hline$\leq 9.9 / \geq 18.1$ & 8 & 6 & & \\
\hline \multicolumn{5}{|l|}{$\operatorname{WBC}^{5}\left(\times 10^{12} / \mathrm{L}\right)$} \\
\hline $4-10$ & 19 & 124 & 2.478 & 0.115 \\
\hline Other & 8 & 25 & & \\
\hline Blood urea nitrogen & $4.33(3.08,7.25)$ & $4.89(4.17,6.00)$ & -0.916 & 0.359 \\
\hline $\mathrm{Na}^{+}$ & $138 \pm 4.44$ & $141.76 \pm 2.75$ & 3.219 & 0.003 \\
\hline \multicolumn{5}{|l|}{$\mathrm{K}^{+}$} \\
\hline $3.5-5.0$ & 23 & 142 & 2.453 & 0.117 \\
\hline Other & 4 & 7 & & \\
\hline \multicolumn{5}{|l|}{ ECG } \\
\hline Normal & 18 & 108 & 2.569 & 0.261 \\
\hline Atrial fibrillation, HR 60-90 & 3 & 6 & & \\
\hline $\begin{array}{l}\text { Abnormal rhythm, premature beat } \geq 5 / \mathrm{min} \text {, abnormal } Q \text { wave and ST/T } \\
\text { wave }\end{array}$ & 6 & 28 & & \\
\hline \multicolumn{5}{|l|}{ Extent of operation } \\
\hline Level 3 & 9 & 99 & 10.569 & 0.001 \\
\hline Level 4 & 18 & 50 & & \\
\hline Total blood loss (ml) & $50(25,200)$ & $10(5,30)$ & -4.376 & $<0.0001$ \\
\hline
\end{tabular}




\begin{tabular}{llll}
\hline No & 20 & 139 & 5.303 \\
\hline Yes & 6 & 20 & 0.021 \\
\hline Malignancy & & 98 & 0.001 \\
\hline No & 7 & 27 & 15.528 \\
\hline Single focus only & 9 & 11 & 12 \\
\hline With lymph node metastasis & 6 & 4 & 98 \\
\hline With distant metastasis & 4 & & \\
\hline
\end{tabular}

Table 5. Assignment of categorical variables in logistic regression analysis for factors influencing postoperative complications after major abdominal surgery.

\begin{tabular}{ll}
\hline Variables & Variable assignment \\
\hline Age & $0=\leq 60,1=61-70,2=71-85$ \\
\hline Pulse rate & $0=50-80,1=$ other \\
\hline Hemoglobin & $0=13-16,1=11.5-12.9 / 16.1-17.0,2=10.1-11.4 / 17.1-18.0,3=\leq 9.9 / \geq 18.1$ \\
\hline Malignant tumor & $0=$ no, $1=$ single focus only $2=$ with lymphatic metastasis, $3=$ with distant metastasis \\
\hline Blood loss & $0=<100,1=101-500,2=501-999,3=\geq 1000$ \\
\hline Extent of operation & $0=$ level $3,1=$ level 4 \\
\hline Abdominal contaminate & $0=$ no, $1=$ yes \\
\hline
\end{tabular}

Table 6. Logistic regression analysis of influencing factors for postoperative complications after major abdominal surgery.

\begin{tabular}{|c|c|c|c|c|c|c|}
\hline Variables & B & S.E. & Wald statistics & $\mathbf{p}$ & OR & $95 \% \mathrm{Cl}$ \\
\hline Frailty & 1.859 & 0.696 & 7.133 & 0.008 & 6.415 & $1.640,25.095$ \\
\hline Blood loss & 2.53 & 0.715 & 12.523 & 0 & 12.553 & $3.092,50.969$ \\
\hline Malignant tumor & 0.652 & 0.302 & 4.645 & 0.031 & 1.918 & $1.061,3.470$ \\
\hline Pulse rate & 2.201 & 0.941 & 5.465 & 0.019 & 9.03 & $1.427,57.141$ \\
\hline Age & 1.669 & 0.717 & 5.419 & 0.02 & 5.308 & $1.302,21.644$ \\
\hline Constant & -4.786 & 0.854 & 31.42 & 0 & 0.008 & \\
\hline
\end{tabular}

Note 1. Model $x^{2}=53.371, p \leq 0.000$. 2. Cox and Snell $R^{2}=0.317$, Nagelkerke $R^{2}=0.546$. 3. Hosmer-Lemeshow test: $x^{2}=7.791, p=0.254>0.05$ is the model that best fit the data.

Table 7. Predictive value of frailty and POSSUM for postoperative complications in patients with major abdominal surgery.

\begin{tabular}{lllllll}
\hline Model & AUC & Z & P & 95\% Cl & Specificity & Sensitivity \\
\hline Frailty score & 0.757 & 4.651 & $<0.0001$ & $00.687,0.818$ & 59.26 \\
\hline POSSUM & 0.777 & 5.462 & $<0.0001$ & $0.696,0.844$ & 94.26 & 51.75 \\
\hline
\end{tabular}

\section{Discussion}

Previous health assessments for the elderly are largely related to comorbidity and the diagnosis of diseases, including cardiovascular disease, cancer and respiratory disorders [8]. In recent years, research on aging has begun to focus on physiological tolerance and frailty as a reflection of the internal environment and functional reserves of patients, which has become an important issue in gerontology research $[15,16]$.
Previous studies have shown that frailty can be used to predict the incidence of adverse outcomes in community populations, and recent studies have shown that it is a good predictor of outcomes for hospitalized patients. Frailty is a useful measurement for the early identification of patients likely to experience adverse outcomes and is useful for promoting early clinical interventions. The frailty phenotype assessment is the most commonly used instrument for frailty evaluation [17]. 
The incidence of frailty is high in elderly patients with major abdominal surgery and frailty screening should be conducted in these patients. In this study, the incidence of frailty in elderly hospitalized patients with abdominal surgery was $18.18 \%$, higher than in previous studies $(10.4 \%-11.3 \%)$. Xi et al. found an $11.1 \%$ incidence of frailty in older populations in communities of Beijing and an incidence of $11.3 \%$ in Taiwan $[18,19]$, reported an incidence of $10.4 \%$ in scheduled surgery patients. In this study, patients unsuitable for frailty assessment due to severe disease were excluded. However, the high chance of frailty in such patients suggests that the actual incidence of frailty in elderly inpatients with major abdominal surgery may be higher than reported in our study. One contribution to the high incidence of frailty in older inpatients with abdominal surgery may be low energy intake; in the elderly; approximately $60 \%$ have an inadequate intake of energy and protein [20]. Our study population included patients with chronic wasting diseases that are often associated with fasting or eating disorders, such as tumors of the digestive system. Decreased energy intake can reduce the resting metabolic rate of patients, resulting in decreased activity. Consequently, energy intake is reduced in the elderly because of energy conservation. Because of this feedback loop, the incidence of frailty in elderly inpatients with abdominal surgery was increased.

Frailty may increase the risk of postoperative complications in elderly patients undergoing major abdominal surgery. A study published in Circulation showed that the median survival rate was low in patients with frailty and that frailty was a risk factor for death during hospital stays and for postoperative complications in cardiac surgery patients [21]. Researchers at Emory University [22], conducted a correlation analysis between frailty and $1 \mathrm{y}$ mortality in patients with abdominal surgery. The latest results show that the mortality of patients with frailty was 6.7 time higher than those with pre-frailty. Adjustments against related factors (age, ASA, CCI, preoperative hemoglobin concentration, GFR, creatinine and albumin concentration) revealed an OR of 3.6 (95\% CI: 0.86-12.46), indicating that frailty was still a risk factor for postoperative complications. Logistic regression analyses in our study showed that frailty was an independent risk factor for postoperative complications, and the risk of postoperative complications for patients with frailty was 6.415 times higher than that of the non-frailty patients, indicating that frailty is a significant risk factor for postoperative complications in elderly patients with major abdominal surgery.

Frailty and POSSUM have the same predictive value. POSSUM is widely applied in surgical fields, and extensive studies have shown that POSSUM effectively predicts the incidence of postoperative complications and death in older patients with abdominal surgery [23-25]. In this study, frailty provided a medium-level predictive value for postoperative complications in elderly patients, comparable to that of POSSUM ( $p>0.05)$. All of the data from this study support the predictive value of frailty, suggesting that frailty assessment results can provide valuable information for clinical decisionmaking. In the context of developing medical technology, the physical condition of the patient is considered more relevant than the operation for the occurrence of postoperative death, after adjustment for the control of variables [12]. Therefore, we believe that frailty could be an effective instrument for preoperative risk assessment to assist in clinical decisionmaking and improve outcomes in elderly patients.

\section{Conclusions}

The rate of postoperative complications in frailty patients is significantly higher compared than that in non-frailty patients. Frailty is an independent risk factor for postoperative complications in elderly patients undergoing major abdominal surgery, who are at the highest relative risk. Frailty has a medium-level predictive value for postoperative complications in elderly patients undergoing major abdominal surgery. Based on the results of this study, medical staff and gerontology researchers can identify and manage frailty in elderly patients to determine risk, aid in preoperative planning and to establish the research foundation for preoperative interventions to reduce the occurrence of postoperative complications and improve outcomes in elderly patients after abdominal surgery. In addition, frailty can be used to identify elderly patients requiring a higher level of postoperative care or to increase the frequency of postoperative visits for these patients.

\section{Relevance to Clinical Practice}

Nurses are responsible for the perioperative care of patients undergoing surgery, and the prevention of postoperative complications is of great significance to patients. However, active nursing measures to prevent postoperative complications are uncommon and nursing has a limited role in the prevention of postoperative complications. Therefore, it is necessary for nurses to perform preoperative risk assessments and to utilize the results of assessments in preoperative interventions. Frailty phenotype evaluation shave many advantages, such as effective risk predictions, ease of use, fast access to data, timeliness and simple environmental requirements, which make it suitable for clinical nurses. Frailty assessment provides a new idea and tools for preoperative risk assessment and perioperative nursing of elderly patients [26].

\section{Source of Funding}

The Programs of the National Natural Science Foundation of China (No. 81272756).

\section{References}

1. National Bureau of Statistics of China. Annual Figures 2014.

2. DeFrances CJ, Podgornik MN. 2004 National Hospital Discharge Survey. Adv Data 2006; 1-19.

3. Cesari M, Onder G, Zamboni V, Manini T, Shorr RI, Russo A, Bernabei R, Pahor M, Landi F. Physical function and self-rated health status as predictors of mortality: results from longitudinal analysis in the ilSIRENTE study. BMC Geriatr 2008; 8: 34. 
4. Pouwels S, Stokmans RA, Willigendael EM, Nienhuijs SW, Rosman C, van Ramshorst B, Teijink JA. Preoperative exercise therapy for elective major abdominal surgery: a systematic review. Int J Surg 2014; 12: 134-140.

5. Hamel MB, Henderson WG, Khuri SF, Daley J. Surgical outcomes for patients aged 80 and older: morbidity and mortality from major noncardiac surgery. J Am Geriatr Soc 2005; 53: 424-429.

6. Riall TS. What is the effect of age on pancreatic resection? Adv Surg 2009; 43: 233-249.

7. Vaupel JW. Biodemography of human ageing. Nature 2010; 464: 536-542.

8. Keevil VL, Romero-Ortuno R. Ageing well: a review of sarcopenia and frailty. Proc Nutr Soc 2015; 74: 337-347.

9. Wells JL, Seabrook JA, Stolee P, Borrie MJ, Knoefel F. State of the art in geriatric rehabilitation. Part II: clinical challenges. Arch Phys Med Rehabil 2003; 84: 898-903.

10. Morley JE, Vellas B, van Kan GA, Anker SD, Bauer JM, Bernabei R, Cesari M, Chumlea WC, Doehner W, Evans J, Fried LP, Guralnik JM, Katz PR, Malmstrom TK, McCarter RJ, Gutierrez RL, Rockwood K, von Haehling S, Vandewoude MF, Walston J. Frailty consensus: a call to action. J Am Med Dir Assoc 2013; 14: 392-397.

11. Chow WB, Rosenthal RA, Merkow RP, Ko CY, Esnaola NF. Optimal preoperative assessment of the geriatric surgical patient: a best practices guideline from the American College of Surgeons National Surgical Quality Improvement Program and the American Geriatrics Society. J Am Coll Surg 2012; 215: 453-466.

12. Story DA. Postoperative mortality and complications. Best Pract Res Clin Anaesthesiol 2011; 25: 319-327.

13. Copeland GP, Jones D, Walters M. POSSUM: a scoring system for surgical audit. Br J Surg 1991; 78: 355-360.

14. Khuri SF, Henderson WG, DePalma RG, Mosca C, Healey NA, Kumbhani DJ. Determinants of long-term survival after major surgery and the adverse effect of postoperative complications. Ann Surg 2005; 242: 326-341.

15. Chang SF, Lin PL. Frail phenotype and mortality prediction: a systematic review and meta-analysis of prospective cohort studies. Int J Nurs Stud 2015; 52: 1362-1374.

16. Revenig LM, Canter DJ, Taylor MD, Tai C, Sweeney JF, Sarmiento JM, Kooby DA, Maithel SK, Master VA, Ogan $\mathrm{K}$. Too frail for surgery? Initial results of a large multidisciplinary prospective study examining preoperative variables predictive of poor surgical outcomes. J Am Coll Surg 2013; 217: 665-670.
17. Buta BJ, Walston JD, Godino JG, Park M, Kalyani RR, Xue QL, Bandeen-Roche K, Varadhan R. Frailty assessment instruments: systematic characterization of the uses and contexts of highly-cited instruments. Ageing Res Rev 2016; 26: 53-61.

18. Chang CI, Chan DC, Kuo KN, Hsiung CA, Chen CY. Prevalence and correlates of geriatric frailty in a northern Taiwan community. J Formos Med Assoc 2011; 110: 247-257.

19. Makary MA, Segev DL, Pronovost PJ, Syin D, BandeenRoche K, Patel P, Takenaga R, Devgan L, Holzmueller CG, Tian J, Fried LP. Frailty as a predictor of surgical outcomes in older patients. J Am Coll Surg 2010; 210: 901-908.

20. Kinney JM. Nutritional frailty, sarcopenia and falls in the elderly. Curr Opin Clin Nutr Metab Care 2004; 7: 15-20.

21. Lee DH, Buth KJ, Martin BJ, Yip AM, Hirsch GM. Frail patients are at increased risk for mortality and prolonged institutional care after cardiac surgery. Circulation 2010; 121: 973-978.

22. Li JL, Henderson MA, Revenig LM, Sweeney JF, Kooby DA, Maithel SK, Master VA, Ogan K. Frailty and one-year mortality in major intra-abdominal operations. J Surg Res 2016; 203: 507-512.

23. Borreman P, De Leyn P, Decaluwé H, Moons J, Van Raemdonck D, Nafteux P, Coosemans W, Lerut $T$. Morbidity and mortality after induction chemotherapy followed by surgery in IIIa-N2 non-small cell lung cancer. Acta Chir Belg 2009; 109: 333-339.

24. Chatterjee AS, Renganathan DN. POSSUM: A scoring system for perforative peritonitis. J Clin Diagn Res 2015; 9: 5-9.

25. Stornes T, Wibe A, Endreseth BH. Complications and risk prediction in treatment of elderly patients with rectal cancer. Int J Colorectal Dis 2016; 31: 87-93.

26. Jiang LL, Ruan H, Xiang XJ, Jia Q. Investigation and analysis of the caring attitude and behaviour of nurses in Shanghai, China. Int J Nurs Pract 2015; 21: 426-432.

\section{${ }^{*}$ Correspondence to}

Binru Han

Xuanwu Hospital

Capital Medical University

PR China 